\title{
Modelling polydisperse nanoparticle production in an enclosed flame spray pyrolysis reactor
}

\author{
Pedro Bianchi Neto*1, Lizoel Buss ${ }^{2,3}$, Udo Fritsching ${ }^{2,3}$, Dirceu Noriler ${ }^{1}$ \\ ${ }^{1}$ Department of Process Engineering - DEPro, University of Campinas, Brazil \\ ${ }^{2}$ Leibniz Institute for Materials Engineering - IWT, Bremen, Germany \\ ${ }^{3}$ Department of Particles and Process Engineering, University of Bremen, Germany \\ *Corresponding author email: pedro.bnchi@hotmail.com
}

\begin{abstract}
In recent years, the scientific interest in population balance models (PBM) is increasing in many research fields, from environmental studies to material production. This technique allows for a precise prediction of population characteristics and behaviour and is a precious ally in process development. Combining PBM and computational fluid dynamics (CFD) has proved very helpful for designing and improving new equipment. That also applies to the production of nanoparticles via the flame spray pyrolysis (FSP) process. The use of reactors with enclosed flames, which allows the fine control of the oxidant in the flame reaction zone, for instance, could benefit from better numerical models for nanomaterial development prediction. In this work, a previously developed model was adapted for the enclosed reactor configuration. In the model, an Eulerian framework is applied to characterize the continuous gas phase, while a Lagrangian framework represents the discrete droplets of the burning spray. A bivariate PBM describes the solid phase, and its polydisperse solution was achieved using the direct quadrature method of moments (DQMoM). Numerical results are validated through comparison with experimental data available in the literature regarding nanoparticle size, presenting good agreement.
\end{abstract}

\section{Keywords}

nanoparticle synthesis; flame spray pyrolysis (FSP); polydisperse modelling; population balance modelling (PBM); DQMoM.

\section{Introduction}

Population balances models (PBM) are commonly used for scientific research when different types of populations must be monitored and their behaviour must be predicted. In the vast majority of cases, however, the studied populations are fully represented by one single variable (univariate cases), e.g., bubble columns and crystallization tanks. In order to make more complex problems approachable, the assumption of drastic simplifications is also usual, what may distance models from reality.

A type of particle population that has been typically modelled with PBMs is the aerosol of nanoparticles formed via flame spray pyrolysis (FSP). In the usual FSP configuration, with an open reactor, a liquid precursor is dissolved in an organic fuel and atomized into fine droplets. These droplets, in turn, evaporate and are immediately ignited due to the thermal energy provided from a pilot flame surrounding the injection region. The organic fuel burns and the combustion reaction produces sufficient energy to enable decomposition and oxidation of the precursor, which results in a supersaturated atmosphere of metallic oxide vapour. This region induces nucleation, forming primary particles that further grow through processes of agglomeration and sintering [1].

Investigations of the FSP process has been expanded to employ more complex reactors and alternative chemical precursors, aiming to improve and design new materials. The addition of an enclosure around the flame is a relatively simple modification, which allows for the control 
of the fuel-oxygen ratio in cases where it is required, e.g., carbon black synthesis [2]. This configuration suppresses the natural air entrainment, resulting in hotter profiles and, consequently, larger primary particle sizes in comparison to the open reactor [3].

Independently of the geometric configuration of the reactor, the FSP process is complex, and involves multiphase highly turbulent flows coupled with mass and heat transfer, chemical reactions and particle formation. Additionally, for such problem, the PBM is typically written in terms of the surface area and volume of the particle agglomerates (bivariate case) to account for the independent variation observed in such variables due to the different phenomena involved. Bivariate cases, however, are considerably harder to solve, and require an adequate method to reach a solution.

Several methods for the solution of PBM have been developed throughout the years, each one best suitable for specific applications. The direct quadrature method of moments (DQMoM), introduced by Marchisio and Fox [4], belongs to the group of quadrature-based methods of moments (QBMM) which apply quadrature formulations as a method of closure for moments of distributions. For bivariate cases, the DQMoM seems ideal as it allows for the relatively simple adaptation from univariate problems and provides precise results at considerably short processing times.

Several studies have modelled the FSP process, including the works from Noriler et al. [5], for pure ethanol burning spray, from Gröhn et al. [6, 7], Mehta et al. [8] and Bianchi Neto et al. [9], for different metallic oxide nanoparticles. In the works of Buss et al. [10, 11], the enclosed configuration has also been modelled. When solving the PBM, however, all the mentioned studies presented monodisperse results, i.e., only in terms of the mean values of the distribution what may mask the function homogeneity, among other characteristics.

In that sense, this study aims to generate a polydisperse solution for the PBM, coupled to computational fluid dynamics (CFD), to represent nanoparticle formation and evolution inside an enclosed FSP reactor, and other aspects of the turbulent reactant flow. For that, the model presented by Bianchi Neto et al. [12] will be applied to the desired geometric configuration. Experimental data presented by Buss et al. [11] are compared to the obtained numerical results for validation.

\section{Mathematical Modeling and Numerical Methods}

The model used to characterize the FSP process combines Eulerian and Lagrangian frameworks under steady conditions, as described by Bianchi Neto et al. [12]. The former is applied to characterize the compressible gas phase as a continuum (considering equations for total mass, momentum, energy, and chemical species). The latter, to characterize the evaporating droplets in the spray as a dispersed phase (considering energy, forces, and mass balances) with two-way coupling between gas and liquid phases. Interphase interaction is taken into account by the drag force, mass and heat transfer. The P1 model is used to compute radiation and the Reynolds-averaged Navier-Stokes (RANS) equations, via the shear-stress transport (SST) $k-\omega$ model, is used to represent turbulence. The eddy dissipation concept (EDC) model accounts for the chemical-turbulence interaction.

Additionally, the nanoparticles (solid phase) are characterized by an Eulerian framework with one-way coupling with the gas phase, given the very low range of the particles Stokes number («1) and their negligible influence on the gas flow. The nucleation of the nanoparticles is computed from the resulting formation rate of the adopted oxide, obtained with the EDC model, and a uniform distribution is assumed for the nuclei. Particle evolution is modelled considering the agglomeration of nanostructures, applying the model proposed by Kruis et al. [13], which is an improvement for non-spherical agglomerates on the Fuch's interpolation kernel, and subsequent sintering, with the model described by Coblenz et al. [14] to compute the characteristic sintering time. Due to the presence of the wall around the aerosol, it is known that thermophoretic deposition occurs, specially regarding the cases with lower rates [11]. The 


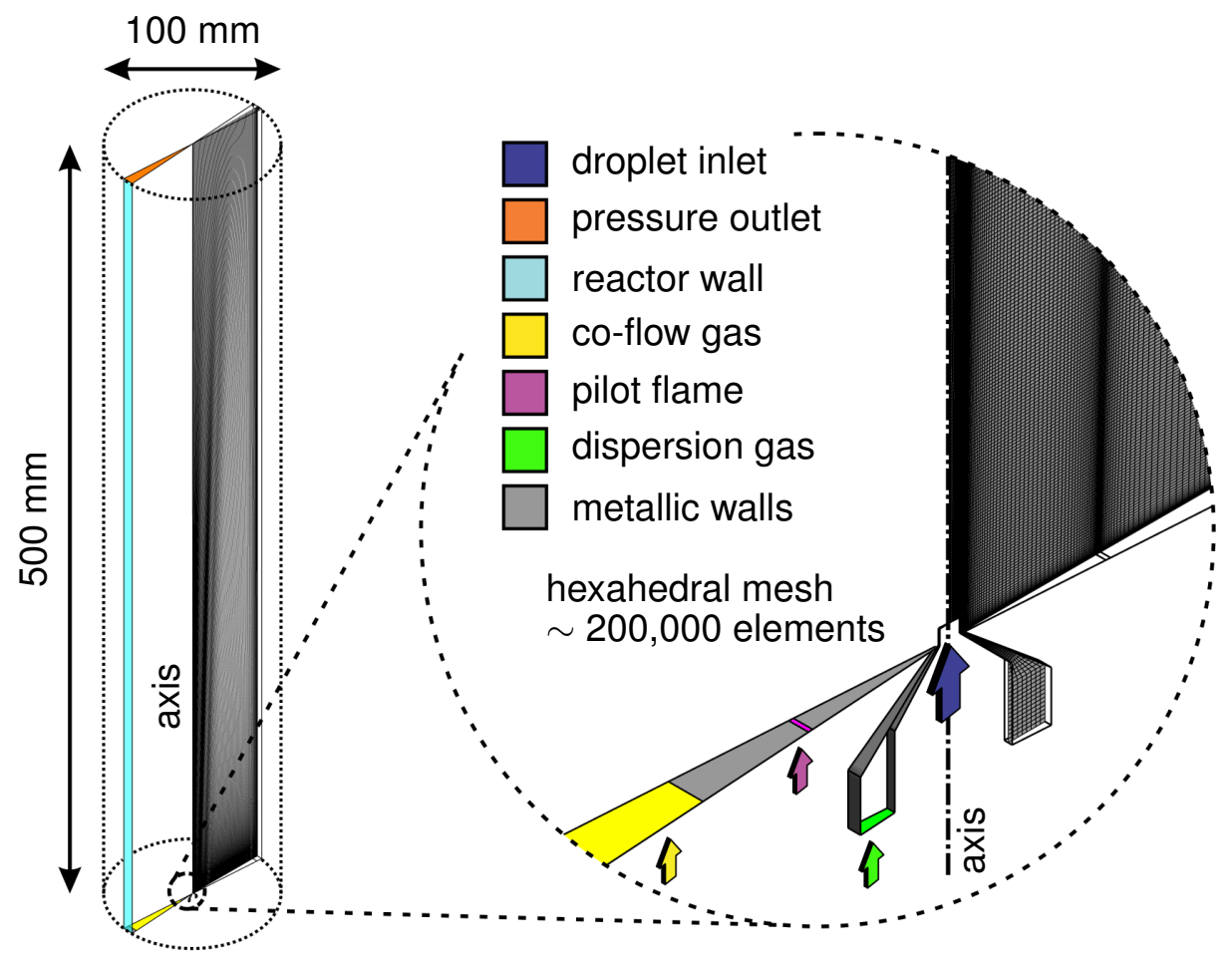

Figure 1. Axisymmetric geometry (with boundary regions) and numerical mesh (with number of elements) adopted in the simulations.

presented model, however, does not yet take into account such effects.

Simulations are carried out with a 2D axisymmetric solver, considering a cylindrical domain with $100 \mathrm{~mm}$ of diameter and $500 \mathrm{~mm}$ of height with the nozzle positioned at its bottom, as shown in Figure 1. It is assumed that the top boundary presents a constant pressure, and the nozzle, which is surrounded by a pilot flame, is located at the centre of a metallic plate. Also, the whole domain is surrounded by a glass cylinder which allows heat transfer (non-adiabatic). A numerical grid with approximately 200,000 hexahedral elements is used for the discretization of the domain, and the evaluation of the reliability of the obtained computational results is verified by the grid convergence index (GCl) method, as presented by Celik et al. [15], presenting an index of less than $0.6 \%$. The simulations were carried out with Ansys Fluent, which uses the finite volume method to solve the partial differential equations of the model. Convergence was considered when the residues for all variables decreased three orders of magnitude.

For the precursor-solvent mixture, a solution of zirconium n-propoxide (IV) in ethanol and npropanol at $0.5 \mathrm{M}\left(72 \% \mathrm{C}_{2} \mathrm{H}_{5} \mathrm{OH}, 8 \% \mathrm{C}_{3} \mathrm{H}_{7} \mathrm{OH}\right.$ and $20 \% \mathrm{C}_{12} \mathrm{H}_{28} \mathrm{O}_{4} \mathrm{Zr}$, in mass $)$ is considered in order to produce nanoparticles of zirconium dioxide. The liquid droplets are injected from a conical surface positioned on top of the nozzle [16], and the initial droplet size distribution is represented by a Rosin-Rammler-Sperling-Bennett (RRSB) function [12]. A mixture of methane and oxygen (19\% and $81 \%$, in mass, respectively) composes the pilot flame, and the chemical reactions associated with the process are considered as presented by Bianchi Neto et al. [12]. Regarding the considered flow rates, the simulated case presents an injection of $5 \mathrm{~mL} / \mathrm{min}$ of the precursor-solvent mixture, dispersed by $5 \mathrm{~L} / \mathrm{min}$ of pure oxygen. A gas co-flow is introduced to the domain in the region surrounding the nozzle at rates of 100 and $400 \mathrm{~L} / \mathrm{min}$ (referred as CF100 and CF400, respectively). The bivariate PBM is solved with the DQMoM, as described in the work of Fox [17], and the source terms of the moments are considered as presented by Zucca et al. [18, 19]. 

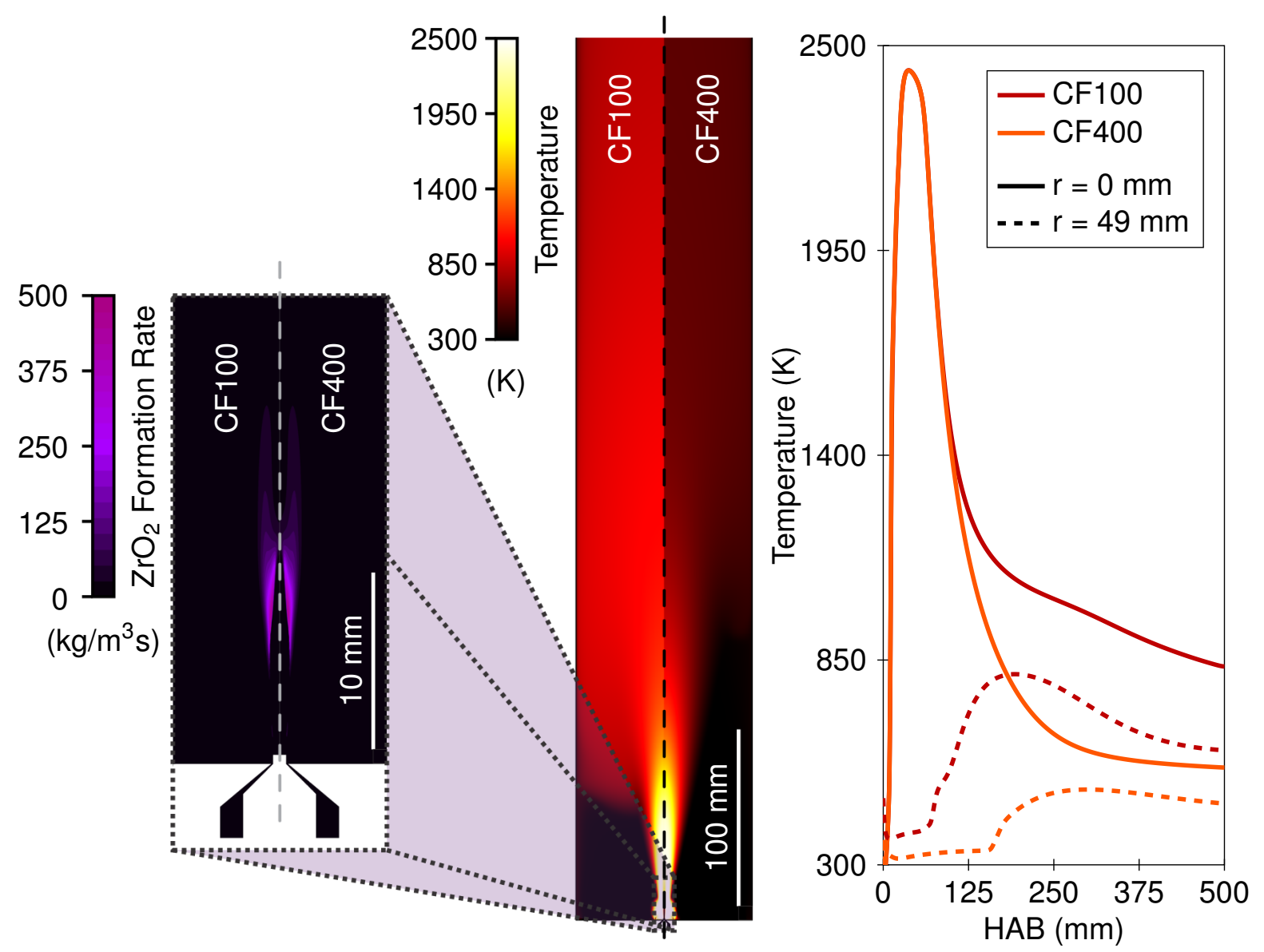

Figure 2. Results of flame temperature for both studied cases: temperature contour and profiles along centre line, $r=0 \mathrm{~mm}$ (solid lines) and near the wall, $r=49 \mathrm{~mm}$ (dashed lines), and contour of $\mathrm{ZrO}_{2}$ formation rate.

\section{Results and Discussion}

Two conditions were studied (CF100 and CF400). For both cases, the solution for the reactive burning spray was obtained, and it was used for the application of the PBM. The results for flame temperature are presented in Figure 2. As shown, the lower co-flow rate hinders the quenching of the system, maintaining higher temperatures throughout the domain. At the lower regions of the reactor, however, very similar temperatures are observed, what results in almost indistinguishable reaction rates. The contour for the $\mathrm{ZrO}_{2}$ formation rate is also shown in Figure 2, and is nearly identical for both cases. As the formation rate of the oxide is used as the boundary condition for the nucleation of the nanoparticles in the PBM, the similarity between both cases should make more evident the influence of the temperature on particle growth. The temperature profiles also indicate a more significant radial gradient at the upper regions of the reactor for the CF100 case.

The results obtained with the solution of the PBM are presented in Figure 3. Given the higher temperatures observed in the case with the lower co-flow rate, larger particle diameters were obtained. The growth of the primary particles occurs mainly due to the sintering of particle agglomerates, which takes place inside the hotter regions. In the profiles displayed in Figure 3, it is noticeable that complete sintering occurs until approximately $125 \mathrm{~mm}$ for the CF400 case. For the CF100 case, however, agglomerates sinter completely until double that height.

Nevertheless, the results drastically overpredict the particle size for the CF100 case compared to experimental values (by over 147\%). For CF400, good agreement is observed (with an error smaller than 3.8\%). The difference observed for the case with the lower co-flow rate can be 

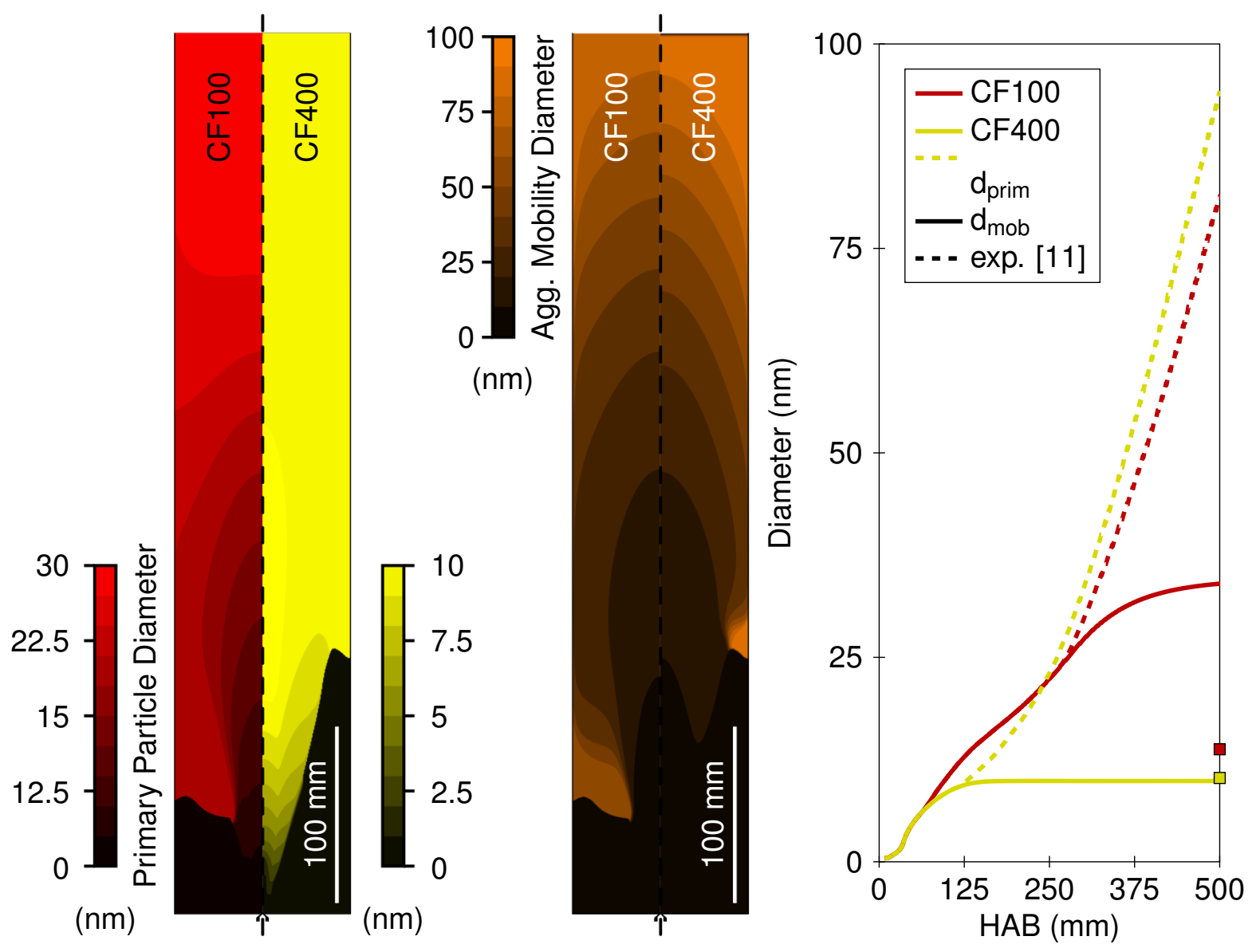

Figure 3. Results of primary particle diameter and agglomerate mobility diameter for both studied case: contour and profiles of radially averaged values, accompanied by experimental results [11] for final primary particle diameter.

linked to the thermophoretic deposition of particles on the reactor walls, which is not considered by the presented model. The lower co-flow rate causes recirculation zones and the particles are trapped continuously, leading to excessive particle growth.

A result from the extended sintering region in the CF100 case is the number of primary particles per agglomerate, as presented in Figure 4. With the complete sintering, smaller agglomerates are found at the top-end of the reactor for such case, whereas, for the CF400 case, as sintering ceases at the lower regions, the primary particles keep agglomerating, adding to their amount in the agglomerates. The characteristic sintering time profiles, also presented in Figure 4, reinforce this effect. It is noticeable that the values rapidly increase for the CF400 case, what indicates a smaller sintering region.

With the polydisperse solution of PBM, it is possible to reconstruct the particle size distributions. For that, the method presented by John et al. [20] was used, assuming a log-normal distribution, as it is typical for aerosols. Figure 5 presents the polydisperse size distribution for both the considered cases, at the FSP reactor top-end.

\section{Summary and Conclusions}

An investigation of a flame spray pyrolysis reactor employed for nanoparticle synthesis was performed at enclosed configuration. Two setups were studied, with 100 and $400 \mathrm{~L} / \mathrm{min}$ of co-flow atmospheric air. Very similar profiles were obtained for both cases at the lower regions of the reactor, closest to the nozzle, including the formation rate of $\mathrm{ZrO}_{2}$, which is used in the calculation of particle nucleation. From approximately HAB (height above burner) $125 \mathrm{~mm}$, however, 


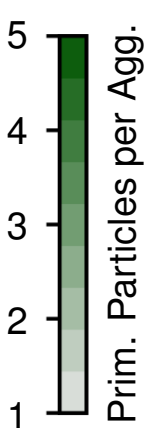

(\#)

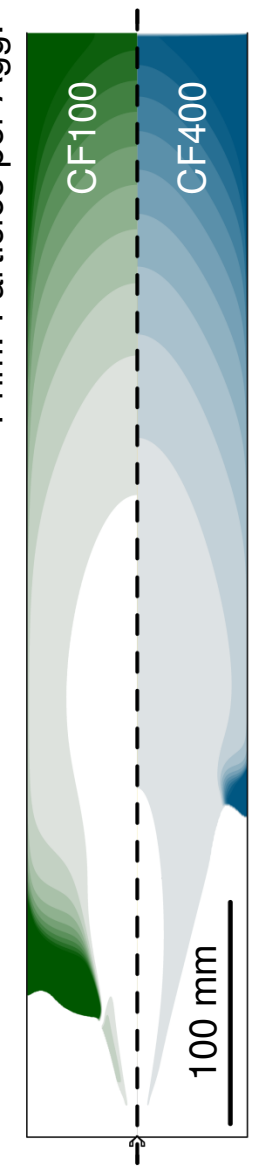

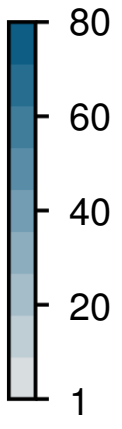

$(\#)$
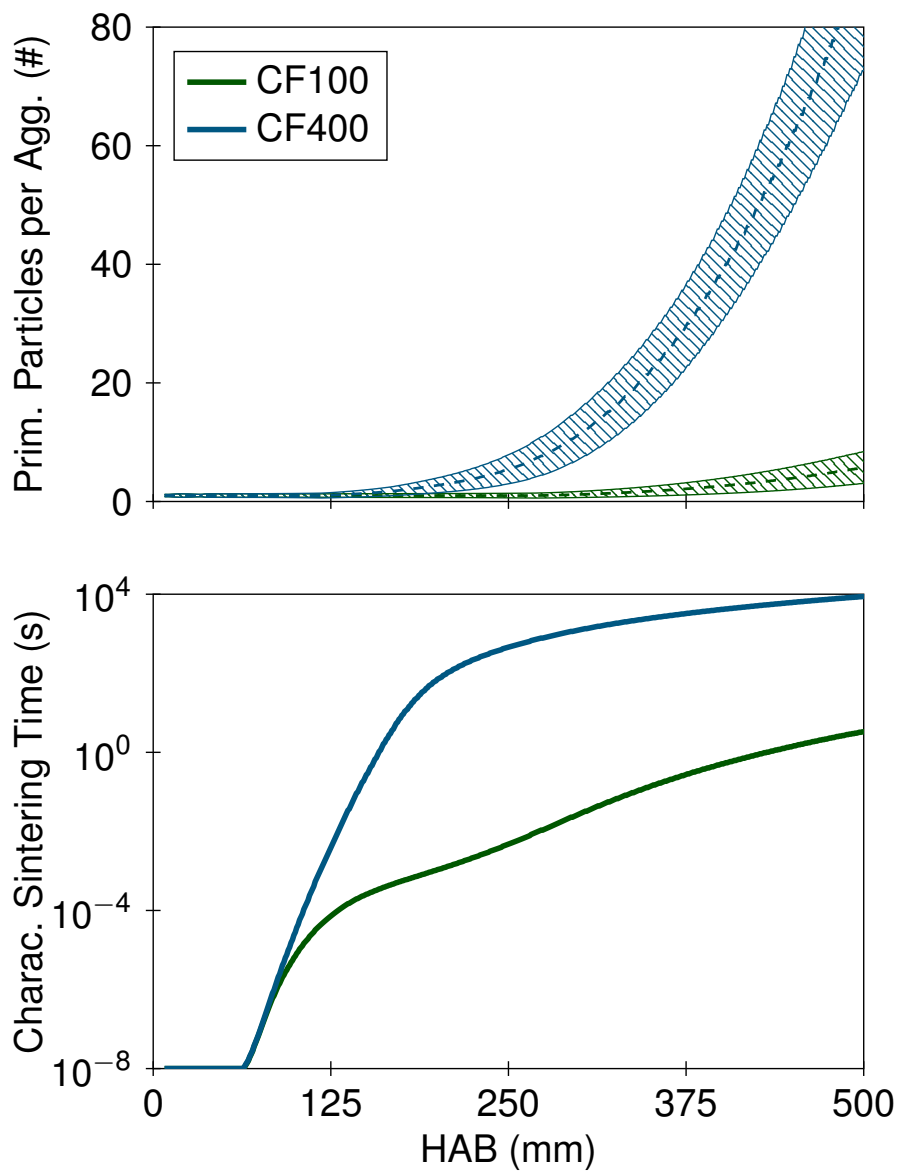

Figure 4. Results of number of primary particles per agglomerate: contour and profiles of radially averaged values (with error region), and radially averaged values of characteristic sintering times along reactor.
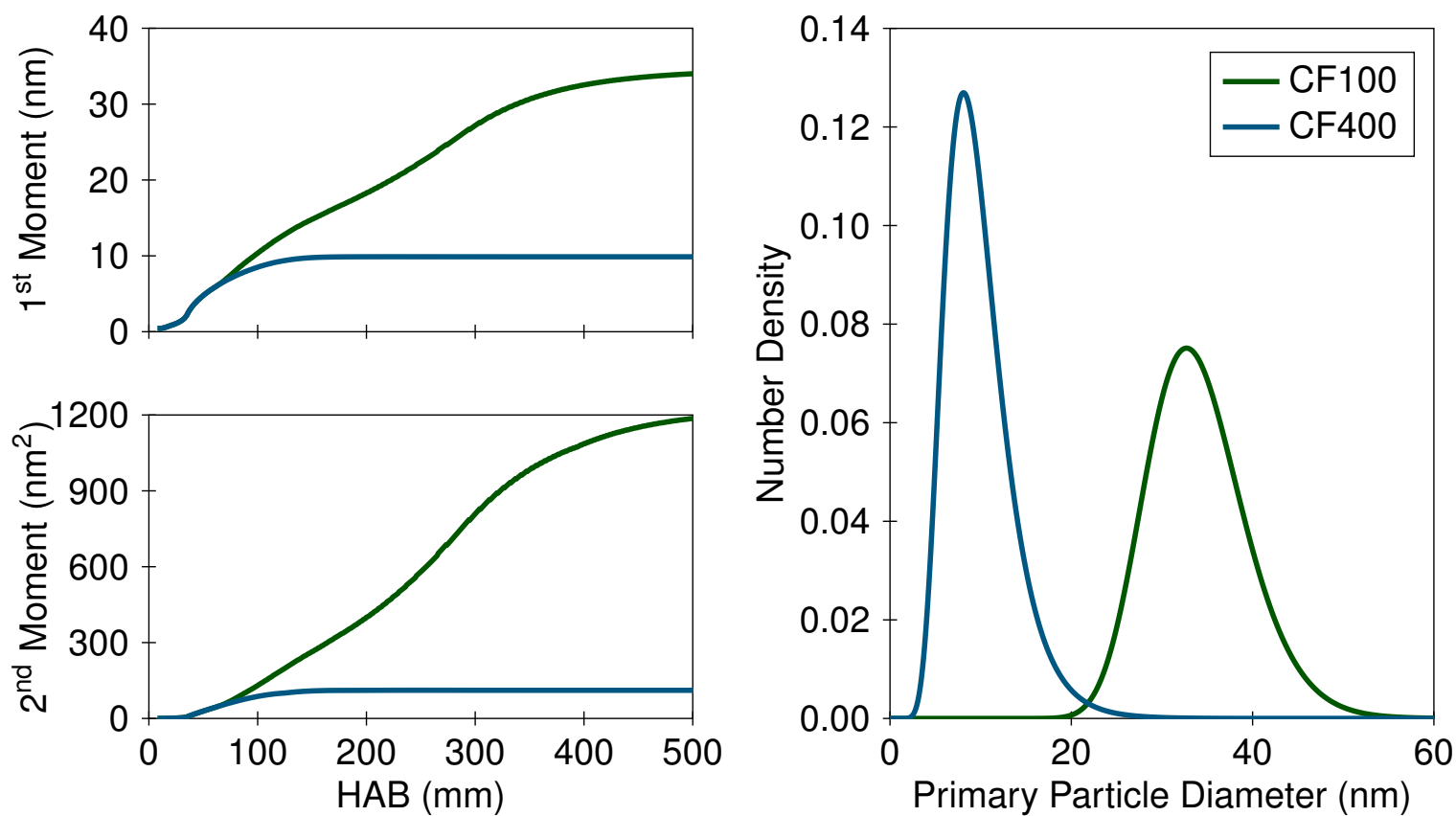

Figure 5. Radially averaged values of the first two raw moments along reactor, and reconstructed size distribution function (log-normal) for nanoparticles at the top-end of the reactor. 
results differ significantly, due to the different co-flow rates. By reducing the co-flow rate, higher temperature profiles and longer residence times are observed inside the reactor. These cause a drastic difference in particle size profiles, given that, due to a larger region of high temperature, the case with the lowest co-flow rate keeps the sintering process for longer. The proposed model overpredicts the final particle size by a factor of 2.47 for the case with co-flow rate of 100 $\mathrm{L} / \mathrm{min}$. For the case using the co-flow rate of $400 \mathrm{~L} / \mathrm{min}$, however, good agreement is observed between numerical and experimental results (lower than 4\%). This difference can be attributed to the thermophoretic deposition of particles on the reactor walls, which was not considered by the model used in this study. Further simulations should be performed, including the deposition phenomenon, in order to improve model predictions. Polydisperse experimental data should also be obtained for further model validation.

\section{Acknowledgements}

This study received funding from the São Paulo Research Foundation - FAPESP (Project ID: 2017/04045-0 and 2020/08502-9), in Brazil. Additional acknowledgement is also directed to the Coordination of Higher Educational Personnel - CAPES (Process ID: BEX 12369/12-8).

\section{References}

[1] Teoh, W. Y., Amal, R., and Mädler, L., 2010, Nanoscale, 8 (2), pp. 1324-1347.

[2] Waser, O., Gröhn, A. J., Eggersdorfer, M. L., and Pratsinis, S. E. , 2014, Aerosol Science and Technology, 48 (11), pp. 1195-1206.

[3] Waser, O., Hess, M., Güntner, A., Novák, P., and Pratsinis, S. E., 2013, Journal of Power Sources , 241, pp. 415-422.

[4] Marchisio, D. L., and Fox, R. O., 2005, Journal of Aerosol Science, 36 (1), pp. 43-73.

[5] Noriler, D., Rosebrock, C. D., Mädler, L., Meier, H. F., and Fritsching, U., 2014, Atomization and Sprays, 24 (6), pp. 495-524.

[6] Gröhn, A. J., Buesser, B., Jokiniemi, J. K., and Pratsinis, S. E., 2011, Industrial and Engineering Chemistry Research, 24 (6), pp. 495-524.

[7] Gröhn, A. J., Pratsinis, S. E., and Wegner, K., 2012, Chemical Engineering Journal, 191, pp. 491-502.

[8] Mehta, M., Fox, R. O., and Pepiot, P, 2015, Industrial \& Engineering Chemistry Research, 54 (20), pp. 5407-5415.

[9] Bianchi Neto, P., Buss, L., Meierhofer, F., Meier, H.F., Fritsching, U., and Noriler, D., 2018, Chemical Engineering and Processing - Process Intensification, 129, pp. 17-27.

[10] Buss, L., Meierhofer, F., Bianchi Neto, P., Meier, H.F. F., Fritsching, U., and Noriler, D., 2018, The Canadian Journal of Chemical Engineering, 9999 (2), pp. 1-12.

[11] Buss, L., Noriler, D., and Fritsching, U., 2020, Flow, Turbulence and Combustion, 105, pp. 1055-1086.

[12] Bianchi Neto, P., Meierhofer, F., Meier, H. F., Fritsching, U., and Noriler, D., 2020, Powder Technology, 370, pp. 116-128.

[13] Kruis, F. E., Kusters, K. A., Pratsinis, S. E., and Scarlett, B., 1993, Aerosol Science and Technology, 19 (4), pp. 514-526.

[14] Coblenz, W. S., Dynys, J. M., Cannon, R. M., and Coble, R. L., 1980, Materials Science Research, 13, pp. 141-157.

[15] Celik, I. B., Ghia, U., Roache, P. J., Freitas, C. J., Coleman, H., and Raad, P. E., 2008, Journal of Fluids Engineering, 130 (7), pp. 1-4.

[16] Bianchi Neto, P., Noriler, D., Meier, H. F., Meierhofer, F., and Fritsching, U., May 22.-27. 2016, 9th International Conference on Multiphase Flow.

[17] Fox, R. O., 2006, Journal of Aerosol Science , 37 (11), pp. 1562-1580. 
[18] Zucca, A., Marchisio, D. L., Barresi, A. A., and Fox, R. O., 2006, Chemical Engineering Science, 61 (1), pp. 87-95.

[19] Zucca, A., Marchisio, D. L., Vanni, M., and Barresi, A. A. , 2007, AlChE Journal, 53 (4), pp. 918-931.

[20] John, V., Angelov, I., Öncül, A. A., and Thévenin, D., 2010, Chemical Engineering Science, 62 (11), pp. 2890-2904. 